\title{
Telepsychiatry or Face-to-face Visit, the Near Paradigm Shift in Psychiatry
}

\section{Hafiz Olatunde, MD, MPH ${ }^{1}$ and Okelue Edwards Okobi, MD, M.Sc. ${ }^{2 *}$}

\author{
${ }^{1} \mathrm{MD}, \mathrm{MPH}$, University of Georgia, Athens, Georgia, USA \\ ${ }^{2}$ MD, M.Sc., Lakeside Medical Center, Belle Glade, Florida, USA \\ E-mail: drhafizolatunde@gmail.com; drokelue.e.okobi@gmail.com \\ *Corresponding author details: Edwards Okobi; drokelue.e.okobi@gmail.com
}

\begin{abstract}
The Covid-19 pandemic, which has necessitated lockdowns because of the ease of virus spread among the populace, especially the unvaccinated, has forced us to reevaluate and reimagine psychiatric care. Before the pandemic telepsychiatry was one of the major ways outpatient visits were carried out. The other being face to face visits. However, there has been an uptick in the use of telepsychiatry for many reasons. This paper explores telepsychiatry, some of its evolutionary factors, the necessary changes that continue to drive the use of telepsychiatry, and its usefulness in certain vulnerable inpatient populations. The incidence and prevalence of mood disorders and anxiety have increased exponentially during the pandemic, and telepsychiatry may have helped make management easier. The use, techniques, and related legal framework of telepsychiatry continue to evolve with advances in technology. The need for regulatory authorities to continue to reform their oversight to accommodate or ensure the safety of this increasing utility of health delivery is crucial, especially at these times.
\end{abstract}

Keywords: covid-19; telepsychiatry; pandemic; psychiatry

\section{SHORT COMMUNICATION}

The COVID-19 pandemic, which brought the world to a universal standstill, again reminds us of the importance of using Telemedicine to deliver care, especially as a means of reducing the risk of cross-contamination caused by close contact [1]. Hence, it is now the primary method of providing care. Telepsychiatry is healthcare delivery between a patient and a provider through video conferencing [2]. The interaction may involve individual, groups, and family therapy, medication management, and patient education. Telemedicine may be a necessity in psychiatry as assessments are dependent on the psychiatric interview and less so on physical examination [3].

To use Telepsychiatry as an effective emergency response, it first needs to become a routinely used part of our health system [1]. This routine use saw an up-tick around the time of the pandemic, on March 11, 2020, when the World Health Organization declared that COVID 19 could be characterized as a pandemic [4]. As of July 1, 2021, The Johns Hopkins Coronavirus Resource center reported that the U.S had over six hundred and five thousand deaths and over 33.7 million confirmed cases of COVID 19 [4]. Telehealth played a significant role in reducing the spread of the virus by limiting face to face contact and viral spread by reinforcing social distancing [1].

To augment care from March 2020, relevant regulations regarding the implementation of Telepsychiatry were enacted and amended, including allowing requirements to travel to originating sites to receive care by U.S. Centers for Medicaid and Medicare [5] services, coverage of visits, suspension of penalties for the usage of non-HIPAA compliant teleconferencing technology like Facetime, Skype, and Zoom. The US. Department of health and Human Services in 2021, declared that they will not impose penalties to health entities for noncompliance if the provision of telemedicine was done in good faith, permitting the use of any public communication method

available for patient interaction [6]. Also, the Ryan Haight exception, that is, the DEA- registered mental health practitioners may issue prescriptions for controlled substances to patients for whom they have not conducted an in-person medical evaluation, provided some necessary conditions are met [5][8].

Studies indicate that the benefits of Telepsychiatry over inperson visit [3] in the delivery of mental health services include improving access for underserved areas of psychiatrist shortage, much- reduced cost of things like the movement of children with intellectual disabilities, improved quality of care like better patient follow up, remote monitoring reduces admissions, and readmissions of severe mental illness, improved engagement due to increased accessibility, reduced risk of viral transmission, reduced burden, and overall patient satisfaction.

Telepsychiatry is not a panacea despite its potential to extend care and improve access to mental health services. Several limitations exist, including maintaining privacy and confidentiality, which can be difficult remotely; breach of data security; and technical failure leading to misinterpretation of relevant interview advice. 
Notwithstanding, there are also legal concerns about loss of patient confidentiality and limited capacity to respond to psychiatric emergencies. There were no published reports of these adverse events yet in the use of Telepsychiatry [3]. For elderly patients with neurocognitive disorders coming from nursing homes, hospital visits may represent a social visit they look forward to, which you may not get in Telepsychiatry. Others include patients with paranoid delusions or those with ideas of reference who already share the notion that they are being monitored with electronic devices may experience an exacerbation of their symptoms even though the majority of the reported cases of the use of videoconferencing in patients with acute paranoid delusions has been positive [7].

Before the pandemic era, there is substantial evidence for the use of Telepsychiatry as a delivery method for mental health services especially in the VA system [3]. Equal now in importance to building a framework for delivering care through Telepsychiatry is a pandemicinformed shift in our approach to patient interactions and treatment recommendations. The COVID-19 pandemic has compelled policymakers, administrators, and providers to challenge both the boundaries of our healthcare system at large and the limiting beliefs around its capacity for rapid, innovative change [3].

\section{REFERENCES}

[1] Smith, A. C., Thomas, E., \& Snoswell, C. L. (2020, June). Telehealth for global emergencies: Implications for coronavirus disease 2019 (COVID-19). PubMed. https://pubmed.ncbi.nlm.nih.gov/32196391/ 26(5):3 09-313

[2] American Psychiatry Association. (2020, August). What is Telepsychiatry? Home | psychiatry.org. https://www.psychiatry.org/patients-families/whatis-telepsychiatry
[3] Hubley, S., Lynch, S. B., \& Schneck, C. (2016, June). Review of key telepsychiatry outcomes. PubMedCentral (PMC). https://www.ncbi.nlm.nih.gov/pmc/articles/P MC4919267/

[4] John Hopkins University of Medicine. (2021, July). CoronaVirus Resource Center. Johns Hopkins Coronavirus Resource Center.

https://coronavirus.jhu.edu/

[5] Kannarkat, Jacob et al. "Mobilization of Telepsychiatry in Response to COVID-19-Moving Toward 21st Century Access to Care" Adm Policy Ment Health. 2020 April 24: 1-3.

[6] The US. Department of Health and Human Services. (2021, January 20). Notification of enforcement discretion for Telehealth. HHS.gov.

https://www.hhs.gov/hipaa/for-

professionals/special-topics/emergency-

preparedness/notification-enforcement-discretiontelehealth/index.html

[7] Sharp, I. R., Kobak, K. A., \& Osman, D. A. (2011). The use of videoconferencing with patients with psychosis: A review of the literature. PubMed Central (PMC). https://www.ncbi.nlm.nih.gov/pmc/articles/PMC310 1132/

[8] Ferrante, T. B., \& Levine, S. J. (2020, March). COVID-19: DEA Confirms Public Health Emergency Exception for Telemedicine Prescribing of Controlled Substances. Attention Required! | Cloudflare.

https://www.foley.com/en/insights/publications/20 20/03/covid19-public-health-exception-telemedicine 